Title

\title{
Children with autism spectrum disorder and comorbid attention deficit hyperactivity
} disorder. Relationships between symptoms and executive function, social cognition, and behavioral problems

\begin{abstract}
Numerous studies have mentioned the importance of discovering the mechanisms underlying the association between autism spectrum disorder (ASD) and comorbid attention deficit hyperactivity disorder (ADHD), with executive function (EF) deficits and theory of mind (ToM) being the most widely investigated cognitive processes. The present study proposed, first, to analyze the executive profile and social cognition processes in children with ASD, ASD+ADHD, ADHD, and typical development (TD). A second objective was to explore the relationship between ASD and ADHD symptoms and EF, ToM, and behavioral problems in children with ASD+ADHD. Participants were 124 children between 7 and 11 years old, distributed in four groups: $37 \mathrm{TD}, 35 \mathrm{ADHD}, 30 \mathrm{ASD}$, and $22 \mathrm{ASD}+\mathrm{ADHD}$, matched on age and IQ. The teachers evaluated the EF with the BRIEF, and the parents assessed the application of ToM skills and the behavioral problems. In addition, a subscale of the NEPSY-II battery was administered to measure performance on emotion recognition. The results showed a similar profile of executive deficits in the ASD+ADHD and ADHD groups, whereas the difficulties in ToM skills in the group with ASD+ADHD were similar to those of the group with ASD. Finally, in children with ASD+ADHD, inattention symptoms were significantly associated with metacognitive deficits and ToM difficulties, and ASD symptoms were associated with behavioral problems. These findings support the need to take inattention symptoms into account and provide training in communication strategies when designing treatments for children with ASD.
\end{abstract}

Key words. ASD+ADHD. Behavioral problems. Comorbidity. Executive functions. Theory of Mind. 


\section{INTRODUCTION}

Autism spectrum disorder (ASD) and attention deficit with hyperactivity and impulsivity

(ADHD) are two of the most common neurodevelopmental disorders. Recently, for the first time, the DSM-5 (American Psychiatric Association (APA), 2013) allowed the joint diagnosis of ASD and ADHD (ASD+ADHD), based on the high comorbidity presented by the two disorders and the co-occurrence of symptoms, especially inattention (Visser et al., 2016). Between 37-85\% of children with ASD are estimated to present comorbid ADHD symptoms (Leitner, 2014), which may be due to common etiological mechanisms. Specifically, the research on psychological processes underlying the ASD+ADHD association has mainly revolved around impairments in executive functioning (EF) and theory of mind (TOM) (Antshell et al., 2016; Doyle et al., 2017), which have been shown to have important effects on social adaptation (Ashwood et al., 2015; Lyall et al., 2017).

The ASD+ADHD group generally shows an executive profile with deficits that are characteristic of both disorders. Specifically, on sustained attention tasks, various studies show worse performance in children with ASD+ADHD, who were similar to the ADHD group in response time variability (Adamo et al., 2014; Lundervold et al., 2016) and attentional orientation impairments (Tye et al., 2014). Moreover, in the ASD+ADHD group, worse performance has been observed on verbal working memory (Andersen et al., 2013; Sinzig et al., 2008b; Takeuchi et al., 2013; Yerys et al., 2009), although no specific deficits have been found in spatial working memory (Gomarus et al., 2009; Sinzig et al., 2008b; Takeuchi et al., 2013). Inhibitory problems seem to be shared by the group with ASD+ADHD and the group with ADHD (Bühler et al., 2011; Chantiluke et al., 2014; Sinzig et al., 2008b; Tye et al., 2014), although sometimes no inhibitory deficits have been found in the comorbid group (Sinzig et al., 2008a; Van der Meer et al., 2012; Yerys et al., 2009). The few studies that have examined the domains of planning and cognitive flexibility indicate a greater planning deficit in the ASD+ADHD group (Pitzianti et al., 2016; Unterrainer et al., 2016), although in some cases the differences were only marginally significant (Colombi et al., 2017). By contrast, no flexibility problems have been observed in the 
comorbid group on laboratory tasks (Sinzig et al., 2008; Van der Meer et al., 2012), and some data even indicate that the presence of ADHD symptoms is associated with better performance on search strategies, which would indicate greater flexibility (Unterrainer et al, 2016). In this context, it is necessary to consider the importance of the assessment methods. For example, difficulties in shifting are not usually found in the ASD+ADHD group, compared to the pure ASD group, on laboratory tasks, but they have been identified using parent reports (Yerys et al., 2009).

A practical question that has aroused interest has to do with the impact of the core ASD and ADHD symptoms on the EF of children who present both disorders. Studies on this topic highlight the relationship between inattention and inhibitory control and verbal working memory problems (Neely et al., 2016; Sinzig et al., 2008b; Takeuchi et al., 2013), as well as stereotyped behavior and flexibility problems (Sinzig et al., 2008b).

The literature on cognition processes, ToM, and emotion recognition (ER) in children with ASD+ADHD, although scarce, suggests that the ADHD symptoms contribute to increasing ToM difficulties in children with ASD, leading to less development of empathy, measured by "Reading the Mind in the Eyes" (Columbi and Ghaziddin, 2017). Likewise, electrophysiological studies have shown atypical processing of the face and gaze in children with ASD and ADHD+ASD, compared to children with ADHD and TD (Groom et al., 2017; Tye et al., 2014; Tye et al., 2014). Increased sustained attention and inhibitory control problems probably worsen the capacity of children with ASD and ASD+ADHD to recognize facial emotions (Sinzig et al., 2008; Van der Meer et al., 2012). Moreover, after controlling reaction time speed, inattention, and inhibition, a greater impairment in affective prosody persists in the ASD+ADHD group (Oerlemans et al., 2014).

The developmental trajectory is an important factor to take into consideration. When comparing children and adolescents with ASD, ADHD, and ASD+ADHD on emotion recognition tasks, differences have been detected between the groups depending on the developmental stage. In the youngest groups (under 10 years old), children with ASD and ASD+ADHD obtain worse performance on the Facial Emotion Matching task than children with ADHD. These results suggest that children with ASD lack 
ToM prerequisites, whereas deficits in mentalist skills in ADHD could develop later, due to their difficulties in social relationships (Bühler et al., 2011).

The adaptive and social functioning of people with ASD are also affected by the inattention and hyperactivity/impulsivity symptomatology (Ashwood et al., 2015; Jang et al, 2013; Lyall et al., 2017; Rao and Landa, 2014; Sikora et al., 2012; Tureck et al., 2013; Yerys et al., 2009). More specifically, the ASD+ADHD group shares emotional and behavioral problems with the ADHD group, but the impairment in adaptive functioning of the ASD group also participates (Craig et al., 2015). The most worrisome data are related to the long-term evolution. Children with ASD without associated manifestations of ADHD experience a reduction in behavior problems over time, whereas in the ASD+ADHD group, the behavioral difficulties remain and even present an incremental trajectory (Flouri et al., 2015).

It is considered a priority to perform an in-depth analysis of the specific profile of each diagnostic group, ASD, ADHD, and, especially, the comorbid group, focusing on estimations of functioning in daily life, in order to design interventions that include strategies that address their specific needs. To this end, the first objective of the present study is to perform a comparison of children with ASD, ASD+ADHD, ADHD, and typical development (TD) on executive processes, social cognition skills, and emotional and behavioral functioning. Based on the results from the majority of the previous studies, we hypothesize that the clinical groups will present impairments compared to the TD group, and, in particular, the group with ASD+ADHD will show an additive deficit of difficulties in inhibition, working memory, and planning (Adamo et al, 2014; Büler et al, 20111; Columbi and Ghaziddin, 2017; Gomarus et al, 2009; Lundervold et al, 2016; Sinzig et al, 2008a; Sinzig et al, 2008b; Takeuchi et al, 2013; Tye et al, 2014; Tye et al, 20016; Unterrainer et el, 2016; Van der Meer et al, 2012), and ToM skills (Bühler et al., 2011; Columbi and Ghaziddin, 2017; Oerlemans et al., 2014; Van der Meer et al, 2012). The second objective is to explore the impact of the core ADHD and ASD symptoms on the EF, social cognition skills, and emotional and behavioral problems of children with ASD+ADHD. We expect that the ADHD symptoms will predict greater impairments in executive functioning, social cognition skills, 
and emotional and behavioral functioning (Jang et al, 2013; Lyall et al., 2017; Neely et al., 2016; Sinzig et al., 2008b; Takeuchi et al., 2013; Tureck et al., 2013).

Unlike in previous studies (Buhler et al, 2011; Columbi and Ghaziddin, 2017; Yerys et al, 2009), all the possible groups are represented, TD, ADHD, ASD, and ASD+ADHD, composed of children with an IQ within the normal range. In addition, except in one isolated study (Yerys et al, 2009), neuropsychological tasks have been used in studies to assess the level of executive functioning performance and social cognition skills. However, given the differences between the demands of a structured evaluation setting and those of the real world, the present study addresses a set of executive, behavioral, and social cognition abilities in daily life, rated by informants who know the subject well, that is, parents and teachers. A final contribution of the present study is the overall, comprehensive nature of the analysis of ASD and ADHD comorbidity, including executive processes, social cognition, and behavior.

\section{METHOD}

\section{Participants}

Participants in this study were 124 children with their families. The children were between 7 and 11 years old and distributed in four groups: 37 children with TD, 35 children with ADHD, and 52 children with ASD, 22 of whom also presented comorbid clinical symptoms with ADHD (ASD+ADHD). The participants had an intellectual capacity (IQ) within the limits of normality (>80), measured with the KBIT (Kaufman and Kaufman, 2000), and they were matched on age and IQ. Moreover, $83.1 \%$ of the participants were boys, and $16.9 \%$ were girls. Language was assessed with the vocabulary subtest of the Wechsler Intelligence Scale for Children - Fourth Edition (WISC-IV) (Wechsler, 2003) because it represents a good measure of the level of general language (Snow et al., 1989) (see table 1). -Insert Table 1-

The participants in the clinical groups had been diagnosed in the Psychiatry and Neuropediatric departments of hospitals and medical centers in the Valencian Community, and they were enrolled in 
public schools. In order to confirm the ADHD diagnosis, the parents and teachers completed the 18 criteria for ADHD from the DSM-5 (APA, 2013). Considering the ratings of the parents and the teachers, $77.1 \%$ of the participants showed a combined presentation, and $22.9 \%$ had a predominance of inattention. The Kappa-Cohen test value was $\kappa=0.97$. In addition, $40 \%$ of the children presented behavior problems, and $71.4 \%$ were taking psycho-medications, mainly psycho-stimulants. To confirm the ASD diagnosis, recommended cut-off points for the Social Communication Questionnaire (SCQ; Rutter et al., 2003a) and the revised autism diagnostic interview (ADI-R; Rutter et al., 2003b) were used, and these instruments were administered by a psychologist from the research team who was accredited in their application. Likewise, to confirm the diagnosis of the 22 children with ASD and clinical symptoms of ADHD, they had to meet the diagnostic criteria for ASD and ADHD. At the time of the assessment, $32.6 \%$ of the children with ASD, with and without comorbid ADHD, were taking psycho-medications (mostly Risperidone and in some cases Methylphenidate) to control behavior problems and irritability. The children with TD were selected in the schools where the clinical sample was obtained. They did not present a history of psychopathologies, and none of them met 6 or more criteria for inattention and hyperactivity/impulsivity from the DSM-5 or the DSM-5 criteria for ASD on the screening carried out before beginning the evaluation.

The exclusion criteria for the children who participated in this study were assessed through an extensive anamnesis carried out previously with the families. They included neurological or genetic diseases, brain lesions, visual, auditory, or motor impairments, and an intelligence quotient below 80 .

\section{Measures}

\section{Executive functioning}

Behavior Rating Inventory of Executive Function (BRIEF; Gioia et al., 2000).

The questionnaire rates the child's executive functions through the teacher's observations of his/her behavior in the school context. It consists of 86 items scored on a Likert-type scale with three response options (never, sometimes, often). The items are grouped in 8 scales that make up three indices. The behavioral regulation index (BRI) determines the child's capacity to change his/her affective state and 
modulate his/her emotions and behavior using appropriate self-control. It contains the subscales of inhibition, shift, and emotional control. The metacognitive index (MI) reflects the child's cognitive capacity to manage tasks and supervise his/her own performance. It includes the subscales of initiation, working memory, planning/organization, organization of materials, and monitoring. Finally, the general executive composite (GEC) is the sum of the two previous indices, the BRI and MI. Direct scores can be transformed into $\mathrm{T}$ scores, with scores equal to or above 65 indicating greater executive problems. In this study, the t scores for the two general BRI and MI indices and their subscales were used. The test-retest reliability of the Spanish adaptation of the teacher version of the BRIEF ranges between .86 and .92 (Maldonado, 2016). These values are similar to those obtained for the original version of the questionnaire (Gioia et al., 2000).

\section{Social Cognition}

Emotion recognition (Developmental Neuropsychological Assessment Battery) (NEPSY II; Korkman et al., 2007).

The NEPSY-II battery contains 32 subtests divided into six domains of cognitive functioning: Attention and executive function, language, memory and learning, sensorimotor area, social perception, and visuospatial processing. The internal reliability coefficients are high $(\mathrm{r} \geq .80)$ (Brooks et al., 2010). For

this study, the emotion regulation subtest from the social perception domain was selected. It is composed of four different areas, and its objective is to rate the ability to recognize the six basic emotions (happy, sadness, fear, anger, neutral, and disgust) based on photographs of children's faces. The response options range from 0 (incorrect response or no response) to 1 (correct response), and the direct scores are converted into scalar scores (mean $10, \mathrm{sd}=3$ ).

Theory of Mind Inventory (ToMI; Hutchins et al., 2014; Spanish adaptation by Pujals et al., 2016). To evaluate the application of ToM skills, the ToMI inventory was completed by the parents. It is composed of 42 items, and each item is an indicator of a specific dimension of Theory of Mind, in order to approach the breadth and complexity of the mentalist skills. The ToMI assesses early skills, such as social references and understanding basic emotions, meta-representations, and second-order inferences, 
on 3 subscales (early, basic, and advanced), and it offers a general average score that was used in this study. Each item is rated from 0 to 20, from "Definitely not" to "Definitely," with a mid-point of "Undecided." Higher scores show the perception of good ToM development.

The ToMI has been widely validated and has good test-retest reliability, internal consistency, and criterion validity in samples with typical development and samples with ASD. It has shown excellent sensitivity (.9) and specificity (.9) when used to examine children with ASD, although its purpose is not to make a differential diagnosis (Hutchins et al., 2012). In the Spanish population, it has also shown high internal consistency using Cronbach's alpha (.96) (Pujals et al., 2016).

\section{Emotional and behavioral functioning}

Strengths and Difficulties Questionnaire (SDQ; Goodman, 1997)

This questionnaire has 25 items and is designed for children from 4 to 16 years old. The items are divided into 5 subscales: Emotional symptoms, Behavioral problems, Hyperactivity, Relationships with peers, and Prosocial behaviors. In addition, it has a total difficulty score obtained by adding together all the subscales except prosocial behavior. The items are scored on a Likert-type scale from 1 (not true) to 3 (completely true). On all the scales, a higher score implies greater difficulty, except the prosocial behavior scale, where a higher score is more positive than a lower one. In this study, the SDQ was filled out by the parents, and the total score on the difficulties scale was used (SDQ Total). In addition, the behavior problems subscale was used to evaluate behavioral difficulties. The SDQ has good statistical and psychometric properties (.73) measured with Cronbach's alpha (Goodman, 2001), and it has shown good reliability in the Spanish population (.76) (Rodríguez et al., 2012).

\section{Procedure}

This study had the approval of the Ethics Committee of the University of Valencia (Helsinki Declaration in the Convention of the European Council, 1964). It also obtained the authorization of the Board of Education of the Valencian Government to approach the schools and the oral and written consent of all the parents of the children included in the study, after informing them about the study objectives. 
The assessment was carried out in specially prepared classrooms in the same schools where the children were enrolled. The parents and children were evaluated during the school day. The tests were administered by experienced professionals who were familiar with the application procedure for the different assessment instruments. In addition, information from teachers was collected in each of the participating schools.

\section{Data analysis}

The statistical analyses were performed with the Statistical Package for the Social Science (SPSS) software, version 22.00 (SPSS Inc., Chicago, IL USA).

After testing the assumptions of the analysis of variance, the differences between the groups with ASD, ADHD, ASD+ADHD, and TD on the scales of executive functioning, social cognition, and SDQ were analyzed. To do so, three multivariate analyses of covariance (MANCOVAs) were conducted, using sex, vocabulary, and the educational level of the parents as covariates. The differences on the tests were verified through covariate analyses of variance (ANCOVAs). For the additional ANCOVAs, the level of significance was established at $\mathrm{p}<.005$ in the comparisons of the different executive functioning domains, after applying the Bonferroni correction, and the value of $\eta^{2}$ p was calculated to test the strength of the association. In a similar way, in the comparison of the social cognition variables (AF, ToMI) and the SDQ, a level of significance of $\mathrm{p}<.01$ was established, after applying the Bonferroni correction. From a dimensional perspective, four multiple linear regression analyses were performed in the comorbid ASD+ADHD group to evaluate the effect of the ASD and inattention and hyperactivity/impulsivity symptoms (independent variables) on the two EF scales (MI and BRI), the total ToMI score, and the total score on the SDQ (dependent variables).

\section{RESULTS}

Comparison of children with $A S D, A S D+A D H D, A D H D$, and $T D$ on executive functioning measures

The MANCOVA carried out to evaluate the main effect of group on the executive functioning indicators was statistically significant [Wilk's Lambda $(\Lambda)=.26, \mathrm{~F}_{(30,317)}=6.20, p<.001, \eta^{2} \mathrm{p}=.36$ ]. The 
confirmation ANCOVAs showed significant differences on Inhibition, $\mathrm{F}_{1,117}=20.13, p<.001, \eta_{\mathrm{p}}^{2}=.34$; shift, $\mathrm{F}_{1,117}=22.18, p<.001, \eta_{\mathrm{p}}^{2}=.36$; Emotional control, $\mathrm{F}_{1,117}=11.81, p<.001, \eta_{\mathrm{p}}^{2}=.23$, and the BRI, $\mathrm{F}_{1,117}=19.84, p<.001, \eta_{\mathrm{p}}^{2}=.33$. The confirmation ANCOVAs also yielded statistically significant differences between the groups on the metacognitive processes: initiative, $\mathrm{F}_{1,117}=23.54, p<.001, \eta_{\mathrm{p}}^{2}=$ .37 ; working memory, $\mathrm{F}_{1,117}=38.50, p<.001, \eta_{\mathrm{p}}^{2}=.49 ;$ planning, $\mathrm{F}_{1,117}=50.28, p<.001, \eta_{\mathrm{p}}^{2}=.56$; organization of material, $\mathrm{F}_{1,117}=27.91, p<.001, \eta_{\mathrm{p}}^{2}=.41 ;$ monitoring, $\mathrm{F}_{1,117}=33.73, p<.001, \eta_{\mathrm{p}}^{2}=.46$ and the total score on the MI, $\mathrm{F}_{1,117}=53.51, p<.001, \eta_{\mathrm{p}}^{2}=.57$. The post hoc analyses revealed significant differences between the TD group and the clinical groups on all the BRIEF variables analyzed in this study, except inhibition and organization of materials, where the TD group and the ASD group showed a significantly lower score than the two groups with ADHD symptoms. Likewise, the ASD group showed a significantly lower score than the two groups with ADHD symptoms (ADHD and ASD+ADHD) on the executive domains of working memory, planning, monitoring, and the two general indexes from the BRI and MI (table 2). The ASD+ADHA group was significantly different from the other two clinical groups because they showed the highest scores on the attention shifting domain. Finally, in the domains of emotional control and initiative, significant differences were only found between the clinical ASD and ADHD groups.

-Insert table 2-

Comparison of children with $A S D, A S D+A D H D, A D H D$, and $T D$ on social cognition measures (emotion recognition and ToMI Inventory)

The MANCOVA performed to evaluate the main effect of group on the social cognition indicators was statistically significant [Wilk's Lambda $(\Lambda)=.27, \mathrm{~F}_{(15,312)}=12.65, p<.001, \eta^{2} \mathrm{p}=.35$ ]. The confirmation ANCOVAs showed significant differences on emotion recognition, $\mathrm{F}_{1,117}=8.21, p<.001, \eta^{2}{ }_{\mathrm{p}}=.17$; ToMI Early, $\mathrm{F}_{1,117}=22.82, p<.001, \eta_{\mathrm{p}}^{2}=.37$; ToMI Basic, $\mathrm{F}_{1,117}=37.46, p<.001, \eta_{\mathrm{p}}^{2}=.49$; ToMI Advanced, $\mathrm{F}_{1,117}=83.94, p<.001, \eta_{\mathrm{p}}^{2}=.68$, and the total score on the ToMI, $\mathrm{F}_{1,117}=62.20, p<.001, \eta_{\mathrm{p}}^{2}=$ .61. Post hoc analyses revealed significant differences between the TD group and the clinical groups on all the variables analyzed. On the AR test, no significant differences were found among the clinical 
groups, unlike on the ToMI, where there were significant differences between the group with ADHD alone and the two groups with ASD symptomatology (ASD and ASD+ADHD) on all the scales and the total score (see table 3).

-Insert table 3-

Comparison of children with $A S D, A S D+A D H D, A D H D$, and TD on emotional and behavioral problems

The MANCOVA performed to evaluate the main effect of group on the SDQ indicators was statistically significant [Wilk's Lambda $(\Lambda)=.22, \mathrm{~F}_{(12,301)}=18.99, p<.001, \eta^{2} \mathrm{p}=.39$ ]. The confirmation ANCOVAs showed significant differences on the scales of emotional symptoms, $\mathrm{F}_{1,117}=12.33, p<.001, \eta_{\mathrm{p}}^{2}=.24$; behavioral problems, $\mathrm{F}_{1,117}=6.61, p<.001, \eta_{\mathrm{p}}^{2}=.14$; hyperactivity, $\mathrm{F}_{1,117}=37.92, p<.001, \eta_{\mathrm{p}}^{2}=.49$; peer problems, $\mathrm{F}_{1,117}=59.96, p<.001, \eta_{\mathrm{p}}^{2}=.60$, and the total score on the SDQ, $\mathrm{F}_{1,117}=42.14, p<.001$, $\eta_{\mathrm{p}}^{2}=.52$. Post hoc analyses revealed significant differences between the TD group and the clinical groups on all the SDQ scales, except the behavioral problems subscale, where the ASD group and the TD group did not present significant differences. Specifically, on hyperactivity the two groups with ADHD were more affected than the ASD group. For peer problems, the two groups with ASD presented higher scores than the group of children with ADHD. The results for the total score on the SDQ revealed significant differences between the group with ASD alone and the ASD+ADHD group (table 4).

-Insert table 4-

Multiple regression analyses measuring the contribution of inattention, $H / I$, and ASD symptoms to EF, ToM, and total $S D Q$ in the comorbid $A S D+A D H D$ group

Four separate multiple regression analyses were carried out to dimensionally explore whether the inattention, H/I, and ASD symptoms are differentially related to the main indices of executive functioning, the ToMI, and the total difficulties on the SDQ in the comorbid ASD+ADHD group. Only two models were significant (see Table 5).

-insert table 5- 
The regressions carried out indicated that the inattention symptoms were a significant individual predictor of both the metacognitive index $(\beta=.40, t=2.3, p=.05)$ and theory of mind $(\beta=-.52, t=-2.7, p=.013)$. All of the indicators together explain 33 and $38 \%$ of the variance, respectively. The ASD symptoms were a significant individual predictor of the total difficulties on the SDQ $(\beta=.53, t=2.7, p=.014)$, with $34 \%$ of the explained variance.

None of the inattention, H/I, or ASD symptoms were significant predictors of the behavioral regulation index (BRI).

\section{DISCUSSION}

The present study aimed to examine the profile of executive functions, social cognition, and behavioral functioning in children with ASD and comorbid ADHD. The first objective was to specifically compare EF, TOM, and behavioral problems in children with ASD, ADHD, ASD+ADHD, and TD. With regard to the executive functioning rated by the teachers, the findings largely confirmed the proposed hypothesis, with the ASD+ADHD group presenting a more pronounced impairment than the ASD group and the TD group. By contrast, the results showed a similar profile between the comorbid group and the ADHD group on the two general indices, BRI and MI, and on the domains of inhibitory control, working memory, planning, organization of materials, and monitoring. The findings are framed within the line of research that shows greater impairment in the executive functioning of children with ASD+ADHD and ADHD alone, compared to children with ASD (Adamo et al., 2014; Bühler et al., 2011; Chantiluke et al., 2014; Lundervold et al., 2016; Sinzig et al., 2008b; Tye et al., 2014). Moreover, the comorbid ADHD symptoms had a greater impact on the attention shifting problems of children with ASD, negatively affecting their flexibility in focusing their attention according to the changing demands of the environment. Similar results were obtained by Yerys et al. (2009) when comparing the EF in children with TD, ASD, and ASD+ADHD, using the parent version of the BRIEF.

In addition, the comparative analyses of the social cognition measures in the four groups revealed the existence of a similar impairment in the groups with ASD (ASD and ASD+ADHD) in their 
capacity to apply ToM skills in social contexts in daily life, thus reinforcing the idea that ToM difficulties are primary deficits in children with ASD (Baron-Cohen et al., 2000). However, no differences in ER were observed among the clinical groups analyzed. These results, similar to those found by Bühler et al. (2011), diverge from other studies that observed greater impairments in facial recognition of emotions in the comorbid group (ASD+ADHD) (Groom et al., 2017; Oerlemans et al., 2014; Sinzig et al., 2008; Tye et al., 2014; Van der Meer et al., 2012). Finally, as in previous studies (Ashwood et al., 2015; Jang et al, 2013; Lyall et al., 2017; Rao and Landa, 2014; Sikora et al., 2012; Tureck et al., 2013; Yerys et al., 2009), the ASD+ADHD group showed more emotional and behavioral difficulties than the two pure clinical groups, which means that the presence of ADHD symptoms worsens the social, adaptive, and behavioral functioning of children with ASD. In summary, the findings suggest the existence of an additive deficit in the comorbid ASD+ADHD group, which shared difficulties with both disorders in executive, cognitive, and social functioning in everyday life.

The second aim of this study was to analyze, from a dimensional perspective, the association between ASD symptoms and ADHD symptoms in the EF, ToM, and behavioral problems of children with ASD. The regression analyses revealed that inattention symptoms had a negative impact on the metacognitive executive processes and ToM skills. Moreover, the ASD symptoms could better explain the more severe social and behavioral problems observed in the comorbid group. The findings are added to the few studies that examined the involvement of ADHD symptoms in executive functioning (Neely et al., 2016; Sinzig et al., 2008b; Takeuchi et al., 2013), finding that inattention symptoms are related to metacognitive processes such as working memory. By contrast, although significant associations have also been found between inhibitory control and inattention symptoms in children with ASD (Neely et al., 2016), our study did not detect relationships between the behavioral regulation index (BRI) and the ADHD symptoms in the comorbid group. In this regard, a possible explanation could be that neuropsychological tasks are usually designed with inhibitory control demands that are different from those found in natural contexts in everyday life. Likewise, the findings support a strong relationship between inattention symptoms and ToM difficulties (Columbi and Ghaziddin, 2017; Sinzig et al., 2008), 
whereas the severity of the autism symptoms would largely explain the adaptive and social problems of children with ASD and comorbid ADHD symptoms (Ashwood et al., 2015).

The present study extends the previous literature by studying a wide range of executive processes, social cognition processes, and behavioral problems in children with ASD+ADHD. Moreover, a more ecological evaluation methodology was implemented in all the processes analyzed. Even so, the study has some limitations. One of them is the low number of participants, especially in the comorbid ASD+ADHD group, which could affect the generalization of the results. In addition, it would be necessary to incorporate longitudinal study designs into the research on the co-occurrence of ASD+ADHD. The evolution of the adaptive and social functioning of children with ASD seems to be determined, in part, by the influence of the comorbid ADHD symptoms, which could worsen the behavior problems (Flouri et al., 2015), and so it would be advisable to examine the directionality of this cooccurrence in the developmental cycle. The research is currently focused on childhood, in spite of evidence that changes occur in the cognitive and social processes throughout development (Hartman et al., 2017). It would be equally important to examine the influence of the different presentations of comorbid ADHD in ASD, especially in terms of the therapeutic approach. Our study suggests that inattention problems would have the most impact on the metacognitive processes and ToM difficulties.

The characteristics that define comorbid ASD+ADHD have important effects on the evaluation and design of treatments. Until now, the topic of comorbid symptoms in ASD has not received enough attention, and so a large percentage of children with ASD+ADHD have not been diagnosed or treated (Joshi et al., 2017), in spite of the negative repercussions of this comorbidity on adaptive and social functioning (Rao and Landa, 2014) and academic capabilities (Lyall et al., 2017). Therefore, programs for children with ASD and symptoms of ADHD must include strategies that strengthen the attentional skills, incorporating them into the treatment from early ages through family collaboration in early intervention programs that specifically target activities to build attentional skills. In a magnificent review article, Mundy (2017) pointed out that task analysis of joint attention suggests that it involves a complex form of social information processing. He highlights the relevance of the emerging neuroscience of joint attention 
in the neurodevelopmental study of ASD, making a compelling case for the argument that joint attention and social-cognitive mentalizing represent a developmentally continuous axis of the cognitive phenotype of ASD.

In later stages of development, a series of programs address a broad range of cognitive functions, including attention and EF for children and adolescents. One example is Attention Improvement Management (AIM) (Sohlberg, Harn, MacPherson, \& Wade, 2014), a 10-week computerized treatment that incorporates goal setting, the use of metacognitive strategies, and computer-based exercises designed to improve various aspects of attention and working memory. Other aids consist of the use of technology by and for adolescents with ASD in school, home, and community settings (Odom et al., 2015). Finally, an individual curriculum adaptation plan is an effective procedure to implement in the school context. Individualizing instructional practices allows personalized academic instruction that is flexible enough to achieve the learning objectives in different academic subjects while taking the needs of students with ASD into account.

\section{References}

Adamo N, Huo L, Adelsberg S, et al. (2014) Response time intra-subject variability: commonalities between children with autism spectrum disorders and children with ADHD. European Child and Adolescent Psychiatry 23: 69-79.

American Psychiatric Association (2013) Diagnostic and Statistical Manual of Mental Disorders: DSM5. Washington, DC: American Psychiatric Association.

Andersen PN, Hovik KT, Skogli EW, et al. (2013) Symptoms of ADHD in children with high-functioning autism are related to impaired verbal working memory and verbal delayed recall. PloS One 8: e64842. 
Antshel KM, Zhang-James Y, Wagner KE, et al. (2016) An update on the comorbidity of ADHD and ASD: A focus on clinical management. Expert Review of Neurotherapeutics 16: 279-293.

Ashwood KL, Tye C, Azadi B, et al. (2015) Brief report: Adaptive functioning in children with ASD, ADHD and ASD+ ADHD. Journal of Autism and Developmental Disorders 45: 2235-2242. Baron-Cohen S, Tager-Flusberg H and Cohen DJ (2000) Understanding Other Minds. Oxford: Oxford University Press.

Brooks B, Sterman E and Strauss E (2010) Test Review: NEPSY-II: A developmental Neuropsychological assessment, Second Ed. Child Neuropsychology 16: 80-101.

Bühler E, Bachmann C, Goyert H, et al. (2011) Differential diagnosis of autism spectrum disorder and attention deficit hyperactivity disorder by means of inhibitory control and 'theory of mind'. Journal of Autism and Developmental Disorders 41: 1718-1726.

Colombi C and Ghaziuddin M (2017) Neuropsychological Characteristics of Children with Mixed Autism and ADHD. Autism Research and Treatment 2017:1-5.

Craig F, Lamanna AL, Margari F, et al (2015). Overlap between autism spectrum disorders and attention deficit hyperactivity disorder: Searching for distinctive/common clinical features. Autism Research, 8, 328-337.

Doyle AE, Vuijk PJ, Doty ND, et al. (2017) Cross-Disorder Cognitive Impairments in Youth Referred for Neuropsychiatric Evaluation. Journal of the International Neuropsychological Society 1-13.

Chantiluke K, Christakou A, Murphy CM, et al. (2014) Disorder-specific functional abnormalities during temporal discounting in youth with Attention Deficit Hyperactivity Disorder (ADHD), Autism and comorbid ADHD and Autism. Psychiatry Research: Neuroimaging 223: 113-120.

Flouri E, Midouhas E, Charman T, et al. (2015) Poverty and the growth of emotional and conduct problems in children with autism with and without comorbid ADHD. Journal of Autism and Developmental Disorders 45: 2928-2938. 
Gioia GA, Isquith PK, Guy SC, et al. (2000) Behavior Rating Inventory of Executive Function. Odessa, FL: Psychological Assessment Resources, Inc.

Gomarus HK, Wijers AA, Minderaa RB, et al. (2009) ERP correlates of selective attention and working memory capacities in children with ADHD and/or PDD-NOS. Clinical Neurophysiology 120: 6072.

Goodman R (1997) The strengths and difficulties questionnaire: A research note. Journal of Child Psychology and Psychiatry 38: 581-586.

Goodman R (2001) Psychometric properties of the strengths and difficulties questionnaire. Journal of the American Academy of Child and Adolescent Psychiatry 40: 1337-1345.

Groom M J, Kochhar P, Hamilton A, et al. (2017) Atypical processing of gaze cues and faces explains comorbidity between autism spectrum disorder (ASD) and attention deficit/hyperactivity disorder (ADHD). Journal of Autism and Developmental Disorders 47: 1496-1509.

Hartman CA, Geurts HM, Franke B, et al. (2016) Changing ASD-ADHD symptom co-occurrence across the lifespan with adolescence as crucial time window: Illustrating the need to go beyond childhood. Neuroscience and Biobehavioral Reviews 71: 529-541.

Hutchins TL, Prelock PA and Bonazinga L (2012) Psychometric evaluation of the theory of mind Inventory (ToMI): A study of typically developing children and children with autism spectrum disorder. Journal of Autism and Developmental Disorders 42: 327-341.

Hutchins TL, Prelock PA and Bonazinga L (2014) Technical Manual for the Theory of Mind Inventory and Theory of Mind Task Battery. Unpublished copyrighted manuscript. http://www.theoryofmindinventory.com.

Jang J, Matson JL, Williams LW, et al. (2013) Rates of comorbid symptoms in children with ASD, ADHD and comorbid ASD and ADHD. Research in Developmental Disabilities, 34: 2369-78.

Joshi G, Faraone SV, Wozniak J, et al. (2017) Symptom profile of ADHD in youth with high-functioning autism spectrum disorder: a comparative study in psychiatrically referred populations. Journal of Attention Disorders 21: 846-855. 
Kaufman AS and Kaufman NI (2000) K-BIT, Test breve de inteligencia de Kauffman [Kaufman Brief Intelligence Test]. Madrid: Pearson.

Korkman M, Kirk U and Kemp S (2007) NEPSY-II: Clinical and Interpretive Manual. San Antonio, TX: The Psychological Corporation (Adaptación española, 2013).

Leitner Y (2014) The co-occurrence of autism and attention deficit hyperactivity disorder in children what do we know? Frontiers in Human Neuroscience 8: 268.

Lundervold AJ, Stickert M, Hysing M, et al. (2016) Attention deficits in children with combined autism and ADHD: a CPT study. Journal of Attention Disorders 20: 599-609.

Lyall K, Schweitzer JB, Schmidt RJ, et al. (2017) Inattention and hyperactivity in association with autism spectrum disorders in the CHARGE study. Research in Autism Spectrum Disorders 35: 1-12.

Maldonado Belmonte MJ (2016) Adaptation of the behavior rating inventory of executive function (BRIEF) to the Spanish population and its usefulness for the diagnosis of attention deficit hyperactivity disorder inattentive and combined subtypes. Doctoral dissertation, Universidad Complutense de Madrid, Spain.

Miranda A, Berenguer C, Roselló B, et al. (2017) Social Cognition in Children with High-Functioning Autism Spectrum Disorder and Attention-Deficit/Hyperactivity Disorder. Associations with Executive Functions. Frontiers in Psychology 8: 1035. Mundy, P. A Review of Joint Attention and Social-Cognitive Brain Systems in Typical Development and Autism Spectrum Disorder. European Journal of Neuroscience. Accepted manuscript online: 18 September 2017.

Mundy P (2017) A Review of Joint Attention and Social-Cognitive Brain Systems in Typical Development and Autism Spectrum Disorder. European Journal of Neuroscience. Accepted Author Manuscript. doi:10.1111/ejn.13720.

Neely RJ, Green JL, Sciberras E, et al. (2016) Relationship Between Executive Functioning and Symptoms of Attention-Deficit/Hyperactivity Disorder and Autism Spectrum Disorder in 6-8 Year Old Children. Journal of Autism and Developmental Disorders 46: 3270-3280. 
Odom SL, Thompson JL, Hedges S, et al. (2015). Technology-Aided Interventions and Instruction for Adolescents with Autism Spectrum Disorder. Journal of Autism and Developmental Disorders 45: 3805-3819.

Oerlemans AM, Van der Meer JM, Van Steijn DJ, et al. (2014) Recognition of facial emotion and affective prosody in children with ASD (+ ADHD) and their unaffected siblings. European Child and Adolescent Psychiatry 23: 257-271.

Pitzianti M, D’Agati E, Pontis M, et al. (2016) Comorbidity of ADHD and high-functioning autism: A pilot study on the utility of the overflow movements measure. Journal of Psychiatric Practice 22: 22-30.

Pujals E, Batlle S, Camprodon E, et al. (2016) Brief report: translation and adaptation of the theory of mind inventory to Spanish. Journal of Autism and Developmental Disorders 46: 685-690.

Rao PA and Landa RJ (2014) Association between severity of behavioral phenotype and comorbid attention deficit hyperactivity disorder symptoms in children with autism spectrum disorders. Autism 18: 272-280.

Rodríguez PJ, Betancort M, Ramírez GM, et al. (2012) Psychometric properties of the parent and ASDcher versions of the strength and difficulties questionnaire (SDQ) in a Spanish sample. International Journal of Clinical Health and Psychology 12: 265-279.

Rutter M, Bailey A and Lord C (2003a) Social Communication Questionnaire. Los Angeles, CA: Western Psychological Services.

Rutter M, Le Couteur A and Lord C (2003b) ADI-R. Autism Diagnostic Interview Revised. Manual. Los Angeles: Western Psychological Services.

Sikora DM, Vora P, Coury DL, et al. (2012) Attention- deficit/hyperactivity disorder symptoms, adaptive functioning, and quality of life in children with autism spectrum disorder. Pediatrics 130: S91S97. 
Sinzig J, Bruning N, Morsch D, et al. (2008a) Attention profiles in autistic children with and without comorbid hyperactivity and attention problems. Acta Neuropsychiatrica 20: 207-215.

Sinzig J, Morsch D, Bruning N, et al. (2008b) Inhibition, flexibility, working memory and planning in autism spectrum disorders with and without comorbid ADHD-symptoms. Child and Adolescent Psychiatry and Mental Health 2: 4.

Sinzig J, Morsch D and Lehmkuhl G (2008) Do hyperactivity, impulsivity and inattention have an impact on the ability of facial affect recognition in children with autism and ADHD? European Child and Adolescent Psychiatry 17: 63-72.

Snow C, Cancino H, Gonzalez P, et al. (1989) Giving formal definitions: an oral language correlate of school literacy. In D. Bloome (Ed.), Classrooms and literacy (pp. 233-249). Norwood, NJ: Ablex.

Sohlberg MM, Harn B, MacPherson H, et al. (2014). Clinical Practice in Pediatric Psychology 2:263280.

Takeuchi A, Ogino T, Hanafusa K, et al. (2013) Inhibitory function and working memory in attention deficit/hyperactivity disorder and pervasive developmental disorders: does a continuous cognitive gradient explain ADHD and PDD traits? Acta Medica Okayama 67: 293-303.

Tureck K, Matson JL, May A, et al. (2013) Externalizing andtantrum behaviours in children with ASD and ADHD compared to children with ADHD. Developmental Neurorehabilitation 16: 52-57.

Tye C, Asherson P, Ashwood KL, et al. (2014) Attention and inhibition in children with ASD, ADHD and co-morbid ASD+ ADHD: an event-related potential study. Psychological Medicine 44: 11011116.

Tye C, Battaglia M, Bertoletti E, et al. (2014) Altered neurophysiological responses to emotional faces discriminate children with ASD, ADHD and ASD+ ADHD. Biological Psychology 103: 125134. 
Unterrainer JM, Rauh R, Rahm B, et al. (2016) Development of Planning in Children with HighFunctioning Autism Spectrum Disorders and/or Attention Deficit/Hyperactivity Disorder. Autism Research 9: 739-751.

Van der Meer JM, Oerlemans AM, van Steijn DJ, et al. (2012) Are autism spectrum disorder and attention-deficit/hyperactivity disorder different manifestations of one overarching disorder? Cognitive and symptom evidence from a clinical and population-based sample. Journal of the American Academy of Child and Adolescent Psychiatry 51: 1160-1172.

Visser JC, Rommelse NN, Greven CU, et al. (2016) Autism spectrum disorder and attentiondeficit/hyperactivity disorder in early childhood: A review of unique and shared characteristics and developmental antecedents. Neuroscience and Biobehavioral Reviews 65: 229-263.

Wechsler D (2003) Wechsler Intelligence Scale for Children: WISC-IV. San Antonio, TX: The Psychological Corporation.

Yerys BE, Wallace GL, Sokoloff JL, et al. (2009) Attention deficit/hyperactivity disorder symptoms moderate cognition and behavior in children with autism spectrum disorders. Autism Research 2: 322-333. 


\section{TABLES}

Table 1. Sample characteristics across the four groups $(\mathrm{N}=124)$

\begin{tabular}{lrrrrrrr}
\hline & TD $(\mathrm{n}=37)$ & ASD $(\mathrm{n}=30)$ & ADHD $(\mathrm{n}=35)$ & ASD $(\mathrm{n}=22)$ & & \\
& Mean (SD) & Mean (SD) & Mean (SD) & Mean (SD) & $\mathrm{F}_{1,120}$ & $\chi^{2}$ & $p$ \\
\hline Age (years) & $8.54(1.2)$ & $8.39(1.3)$ & $9.14(1.4)$ & $8.86(1.3)$ & 2.00 & - & .118 \\
IQ & $102.11(8.9)$ & $100.37(12.4)$ & $99.03(9.8)$ & $102.86(13.0)$ & .75 & - & .521 \\
Vocabulary & $12.91(2.7)$ & $11.56(3.3)$ & $10.14(2.2)$ & $11.45(3.4)$ & 5.43 & - & $.002^{*}$ \\
Parental education & $3.5(.96)$ & $3.4(1.0)$ & $1.8(1.4)$ & $3.1(1.2)$ & 14.65 & - & $.000^{*}$ \\
ASD Symptoms & - & $15.70(3.4)$ & - & $16.36(2.7)$ & & & \\
Inattention & $6.83(5.0)$ & $22.96(6.5)$ & $41.31(6.8)$ & $37.59(6.2)$ & 218.0 & - & $.000^{*}$ \\
H/I & $5.83(4.6)$ & $15.82(9.5)$ & $31.54(10.1)$ & $25.59(8.3)$ & 63.1 & - & $.000^{*}$ \\
Sex (\% males) & $62.1 \%$ & $90.0 \%$ & $91.4 \%$ & $95.4 \%$ & - & 16.65 & $.001 *$ \\
Medication (\% yes) & $0.0 \%$ & $26.6 \%$ & $71.4 \%$ & $40.9 \%$ & - & 42.17 & $.000^{*}$ \\
\hline
\end{tabular}

ASD+: ASD+ADHD; Inattention: Inattention DSM 5; H/I: Hyperactivity/Impulsivity DSM 5; ASD Sympt: Autism Symptoms DSM 5; Parental education was measured as highest level of mother or father $(0=$ elementary school, 1 = Compulsory secondary school, $2=$ Medium level vocational training, $3=$ Upper secondary education (Bachiller) or Superior level vocational training, 4= University degree.

$* p<.05$ 
Table 2. Differences between groups on executive functions

\begin{tabular}{|c|c|c|c|c|c|c|c|c|c|}
\hline \multirow[b]{2}{*}{$\mathrm{EF}$} & \multicolumn{2}{|c|}{$\begin{array}{c}1 . \mathrm{TD} \\
(\mathrm{n}=37)\end{array}$} & \multicolumn{2}{|c|}{$\begin{array}{l}\text { 2.ASD } \\
(\mathrm{n}=30)\end{array}$} & \multicolumn{2}{|c|}{$\begin{array}{l}\text { 3.ADHD } \\
(\mathrm{n}=35)\end{array}$} & \multicolumn{2}{|c|}{$\begin{array}{l}\text { 4.ASD+ADHD } \\
\quad(\mathrm{n}=22)\end{array}$} & \multirow[b]{2}{*}{ Post hoc } \\
\hline & $\mathrm{M}$ & SD & $\mathrm{M}$ & SD & $\mathrm{M}$ & SD & $\mathrm{M}$ & SD & \\
\hline Inhibit & 48.54 & 7.56 & 54.27 & 12.50 & 69.97 & 12.03 & 63.09 & 11.58 & $1,2<3,4$ \\
\hline Shift & 51.84 & 9.33 & 64.37 & 12.51 & 67.43 & 12.36 & 75.77 & 11.59 & $1<2,3,4 ; 2,3<4$ \\
\hline Emotional C & 50.08 & 7.01 & 60.87 & 14.92 & 71.89 & 15.29 & 67.18 & 14.80 & $1<2,3,4 ; 2<3$ \\
\hline BRI & 49.65 & 6.49 & 60.20 & 14.11 & 70.86 & 12.65 & 70.14 & 12.04 & $1<2,3,4 ; 2<3,4$ \\
\hline Initiate & 50.43 & 9.60 & 60.10 & 8.65 & 68.51 & 9.98 & 66.96 & 9.04 & $1<2,3,4 ; 2<3$ \\
\hline WM & 47.68 & 8.39 & 55.83 & 8.78 & 73.09 & 12.92 & 69.41 & 12.25 & $1<2,3,4 ; 2<3,4$ \\
\hline Plan & 46.89 & 6.43 & 56.33 & 9.09 & 71.09 & 10.76 & 69.05 & 9.63 & $1<2,3,4 ; 2<3,4$ \\
\hline O. Materials & 47.65 & 4.27 & 53.67 & 8.70 & 67.49 & 13.88 & 68.05 & 10.97 & $1,2<3,4$ \\
\hline Monitor & 48.14 & 7.50 & 58.27 & 10.88 & 72.46 & 10.51 & 68.14 & 8.49 & $1<2,3,4 ; 2<3,4$ \\
\hline MI & 47.78 & 6.60 & 57.37 & 8.20 & 73.54 & 11.46 & 70.05 & 8.44 & $1<2,3,4 ; 2<3,4$ \\
\hline
\end{tabular}

Emotional C: Emotional control; WM: Working memory; O. Material: Organization of Materials; BRI: Behavioral Regulation Index; MI: Metacognition Index $* p<.005$ (Bonferroni correction) 
Table 3. Differences between groups on ER and Theory of Mind

\begin{tabular}{lccrrrrrrr}
\hline & \multicolumn{2}{c}{$\begin{array}{c}1 . T D \\
(\mathrm{n}=37)\end{array}$} & \multicolumn{2}{c}{$\begin{array}{c}2 . \text { ASD } \\
(\mathrm{n}=30)\end{array}$} & \multicolumn{2}{c}{$\begin{array}{c}\text { 3.ADHD } \\
(\mathrm{n}=35)\end{array}$} & $\begin{array}{c}4 . \text { ASD+ADHD } \\
(\mathrm{n}=22)\end{array}$ & \\
\hline & $\mathrm{M}$ & $\mathrm{SD}$ & \multicolumn{1}{c}{$\mathrm{M}$} & \multicolumn{1}{c}{ SD } & \multicolumn{1}{c}{$\mathrm{M}$} & $\mathrm{SD}$ & $\mathrm{M}$ & $\mathrm{SD}$ & Post hoc \\
\hline AR & 28.41 & 2.73 & 23.43 & 4.12 & 24.11 & 4.38 & 24.18 & 4.13 & $1>2,3,4$ \\
ToMI Early & 18.98 & 1.13 & 14.89 & 3.13 & 17.22 & 1.83 & 14.01 & 3.72 & $1>2,3,4 ; 3>2,4$ \\
ToMI Basic & 19.03 & 1.12 & 13.33 & 3.29 & 16.68 & 2.14 & 12.35 & 3.40 & $1>2,3,4 ; 3>2,4$ \\
ToMI Adv & 17.24 & 2.36 & 8.77 & 3.18 & 14.13 & 3.05 & 7.00 & 2.47 & $1>2,3,4 ; 3>2,4$ \\
ToMI Total & 18.38 & 1.42 & 12.04 & 2.86 & 15.87 & 2.13 & 10.78 & 2.82 & $1>2,3,4 ; 3>2,4$ \\
\hline
\end{tabular}

ER: Emotion recognition; ToMI: Theory of mind inventory total score; ToMI Adv: Theory of mind advanced

$* p<.01$ (Bonferroni correction) 
Table 4. Differences between groups on Strengths and Difficulties Questionnaire

\begin{tabular}{lrrrrrrrrr}
\hline & \multicolumn{2}{c}{$\begin{array}{c}1 . T D \\
(\mathrm{n}=37)\end{array}$} & \multicolumn{2}{c}{$\begin{array}{c}2 . \text { ASD } \\
(\mathrm{n}=30)\end{array}$} & \multicolumn{2}{c}{$\begin{array}{c}\text { 3.ADHD } \\
(\mathrm{n}=35)\end{array}$} & $\begin{array}{c}4 . \text { ASD+ADHD } \\
(\mathrm{n}=22)\end{array}$ & \\
\hline & $\mathrm{M}$ & $\mathrm{SD}$ & $\mathrm{M}$ & $\mathrm{SD}$ & $\mathrm{M}$ & $\mathrm{SD}$ & $\mathrm{M}$ & $\mathrm{SD}$ & Post hoc \\
\hline Emotional & 1.64 & 1.25 & 4.23 & 1.90 & 4.57 & 2.45 & 5.04 & 2.55 & $1<2,3,4$ \\
Behavioral & 1.29 & 1.35 & 2.43 & 1.73 & 4.11 & 2.52 & 3.77 & 2.02 & $1<3,4 ; 2<3$ \\
Hyperactivity & 2.08 & 1.84 & 5.13 & 2.66 & 8.22 & 1.92 & 7.86 & 1.88 & $1<2,3,4 ; 2<3,4$ \\
Peer problems & .40 & .83 & 5.63 & 2.25 & 2.88 & 1.90 & 5.95 & 1.98 & $1<2,3,4 ; 3<2,4$ \\
SDQ Tot & 5.43 & 3.48 & 17.43 & 6.04 & 19.80 & 6.87 & 22.63 & 5.24 & $1<2,3,4 ; 2<4$ \\
\hline
\end{tabular}

Emotional: Emotional symptoms scale; Behavioral: Behavioral problems scale; Hyperactivity: Hyperactivity scale; Peer problems: Peer problems scale; SDQ Tot (Strengths and Difficulties Questionnaire total difficulties scale)

$* p<.01$ (Bonferroni correction) 
Table 5. Multiple regression analysis for inattention, hyperactivity/impulsivity, and ASD symptom scales predicting executive functions, theory of mind, and behavioral problems in comorbid ASD+ADHD Group

\begin{tabular}{lcccc}
\hline & $B$ & $S E$ & $\beta$ & $t$ \\
\hline EF-BRI & \multicolumn{2}{l}{$F(3,18)=.86 ; R^{2}=.12$} & \\
Inattention & .22 & .42 & .11 & .52 \\
H/I & .39 & .32 & .27 & 1.21 \\
ASD Symptoms & .67 & .98 & .15 & .68 \\
\hline EF-MI & $F(3,18)=2.91 ; R^{2}=.32$ \\
Inattention & .53 & .26 & .40 & $2.33^{*}$ \\
H/I & .15 & .19 & .15 & .79 \\
ASD Symptoms & 1.05 & .60 & .33 & 1.73 \\
\hline ToM & $F(3,18)=3.67^{*} ; R^{2}=.38$ \\
Inattention & -.23 & .08 & -.52 & $-2.76^{*}$ \\
H/I & -.01 & .06 & -.04 & -.22 \\
ASD Symptoms & -.30 & .19 & -.29 & -1.55 \\
\hline SDQ-Tot & $F(3,18)=3.11^{*} ; R^{2}=.34$ & .19 \\
Inattention & .15 & .16 & .18 & .93 \\
H/I & .09 & .12 & .14 & .73 \\
ASD Symptoms & 1.09 & .37 & .53 & $2.73 *$ \\
\hline
\end{tabular}

Inattention DSM 5; H/I: Hyperactivity/Impulsivity DSM 5; ASD Sympt: Autism spectrum disorder symptoms-DSM 5; BRI: Behavioral regulation index; MI: Metacognition index; ToM: Theory of mind inventory; SDQ: Social difficulties questionnaire total score $* p<.05$ 